EXTENDED REPORT

\title{
The relation between disease asymmetry and severity in keratoconus
}

\author{
J J Nichols, K Steger-May, T B Edrington, K Zadnik, the CLEK Study Group
}

Br J Ophthalmol 2004;88:788-791. doi: 10.1136/bjo.2003.034520

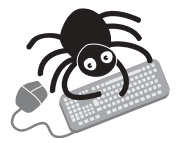

A complete list of the CLEK Study Group members can be found on the BJO website (www.bjophthalmol. com)

See end of article for authors' affiliations

.....................

Correspondence to: K Zadnik, OD PhD, OSU

College of Optometry, 320

West 10th Avenue, PO

Box 182342, Columbus,

$\mathrm{OH} 43210$, USA.

Zadnik.4@osu.edu

Accepted

4 November 2003
Background/aim: Keratoconus is described as a bilateral, asymmetric, non-inflammatory corneal ectasia. The purpose of the study was to examine the relation between disease asymmetry and severity in the Collaborative Longitudinal Evaluation of Keratoconus (CLEK) Study cohort.

Methods: Analyses included 1037 keratoconus patients from the CLEK Study baseline examination visit, none of whom had undergone corneal surgery in either eye. Disease asymmetry was determined by taking the difference between eyes for continuous variables. For categorical variables, asymmetry was categorised by whether the variable was present in neither, one, or both eyes. Disease severity was defined using the first definite apical clearance lens (FDACL) technique (a rigid contact lens to measure corneal curvature) from the worse (steeper) eye. Statistical analyses included Pearson's correlation coefficients (continuous variables) and analysis of variance (categorical variables).

Results: There were generally weak correlations between asymmetry and severity for low contrast habitual visual acuity $(r=0.12, p=0.0003)$, high contrast habitual visual acuity $(r=0.14, p<0.0001)$, low contrast best corrected visual acuity $(r=0.21, p<0.0001)$, and high contrast best corrected visual acuity $(r=0.29$, $p<0.0001)$. Asymmetry in refractive error was more moderately correlated with disease severity $(r=0.41$, $p<0.0001)$, as was asymmetry in the flat $(r=0.61, p<0.0001)$ and steep keratometric readings $(r=0.54$, $\mathrm{p}<0.0001)$. The average FDACL was significantly steeper in patients who had one eye with Vogt's striae, Fleischer's ring, or corneal scarring compared with the average FDACL when neither eye had these findings.

Conclusion: Keratoconus patients with more severe disease are also more asymmetric in their disease status.

$$
l_{\text {int }}^{\mathrm{s}}
$$
previous studies, inferences regarding the clinical asymmetry of keratoconus have been made by reporting the frequency of unilateral cases of disease or unilateral corneal transplants associated with keratoconus. ${ }^{1-5}$ Recently, we reported on the between eye asymmetry associated with keratoconus and compared the degree of asymmetry between the "better" and "worse" eyes with a retrospectively assembled group of myopic subjects. ${ }^{6}$ Large between-eye differences were found in high and low contrast visual acuity, refractive error, keratometry, and the first definite apical clearance lens (FDACL) measure of corneal curvature, especially compared with the normal myopic subjects.

To our knowledge, there have been no reports relating the degree of asymmetry in keratoconus to the severity of the disease. Often at the time of diagnosis of keratoconus, one eye is primarily affected, showing clinically measurable signs of the disease, while the other eye remains clear of these signs for several years. In other cases, larger degrees of between-eye asymmetry in keratoconus seem to be associated with more severe cases of the disease. The purpose of this report is to describe the relation between disease asymmetry and severity in the Collaborative Longitudinal Evaluation of Keratoconus (CLEK) Study cohort.

\section{METHODS}

\section{CLEK study patients}

Baseline eligibility criteria included age (at least 12 years old), irregular corneal surface (keratometry distortion, scissoring of the retinoscopy reflex, or an irregular ophthalmoscopic red reflex in either eye), the presence of a biomicroscopic sign associated with keratoconus (Vogt's striae, Fleischer's ring, or corneal scarring in either eye), and the self reported ability to complete CLEK study examinations for at least three additional years. Patients were not included if they had bilateral corneal transplants or if they had non-keratoconic ocular disease in both eyes (cataract or retinal disease in both eyes). There were 1209 patients enrolled between 31 May 1995 and 29 June 1996. At the baseline examination, all patients signed informed consent documentation regarding their participation in the study, which was approved by each site specific institutional review board. Baseline data were used for all analyses described below.

\section{CLEK Study clinical measures}

We briefly report on those measures relevant to these analyses as our methods have been previously published..$^{6-10}$

Visual acuity, keratometry, and manifest refraction High and low contrast visual acuity was measured using the Bailey-Lovie visual acuity charts with habitual correction and with best correction (with spherocylindrical overrefraction for contact lens wearers and with a CLEK Study rigid contact lens and overrefraction for those subjects not wearing their own contact lenses). ${ }^{8}$ Each eye was tested independently for habitual and best corrected visual acuity. The total number of letters correctly identified was recorded (out of 70 letters).

Manifest refraction was measured using standard clinical techniques. Larger lens power and cylindrical axis alternative measurement methods were used when patients had extremely poor vision.

Abbreviations: CLEK, Collaborative Longitudinal Evaluation of Keratocomus; FDACL, first definite apical clearance lens. 
Keratometry was used to measure the corneal power in the flat and steep meridians of both eyes two consecutive times, and these measures were averaged. Additional lenses $(+1.25 \mathrm{D}$ or $+2.25 \mathrm{D})$ were used to extend the range of readings up to $68.30 \mathrm{D}$ as needed. ${ }^{11}$

\section{Slit lamp biomicroscopy and photography}

Outcomes assessed using the slit lamp that are pertinent to these analyses included the presence or absence of Vogt's striae, Fleischer's ring, and corneal scarring. For corneal scarring, the clinician was to record the location and density of the scar, and to state the presence of the scar in the following format: definitely not scarred, probably not scarred, probably scarred, and definitely scarred. Parallelepiped and oblique corneal photographs were taken, and undeveloped film was sent to the CLEK Photography Reading Center at the Ohio State University for processing and labelling. A masked reader then assessed the photograph using the same grading scheme. For this report, the cornea was considered scarred at baseline if both the clinician and reader characterised the scar as "definitely scarred" and the scar was present in the central $6 \mathrm{~mm}$ of the cornea.

\section{FDACL}

The FDACL technique with a series of rigid contact lenses was used to measure corneal curvature. ${ }^{7}$ The FDACL procedure was developed to measure severity of keratoconus, and the ultimate goal of the FDACL technique is to measure the sagittal height of the cone apex. The technique allows for corneal curvature measures between $42.19 \mathrm{D}$ and $67.50 \mathrm{D}$ and is more robust and reliable than keratometry or videokeratography. ${ }^{7}$ The procedure is stopped when the flattest lens in the trial set that showed definite apical clearance was established. This is the FDACL, and after completing this sequence, a lens $0.2 \mathrm{~mm}$ flatter than FDACL was placed on the eye and photographed. The film was sent to the CLEK Photography Reading Center for processing, labelling, and reading by independent, masked readers.

\section{Data management and statistical analyses}

All analyses were conducted using the Statistical Analysis System (SAS version 8.2, Carey, NC, USA) at the Coordinating Center located in the Division of Biostatistics of Washington University, St Louis, MO, USA. Patients were excluded from these analyses if they had undergone corneal surgery in either eye (penetrating keratoplasty or epikeratoplasty), or if they were wearing monovision contact lens correction. Patients were further excluded from the analysis of a specific outcome if they had missing values for that outcome or FDACL. For patients whose visual acuity was tested at $1 \mathrm{~m}$ in either eye, 30 letters (because of the six logarithmic unit difference between test distances) were subtracted from that eye in order to convert the acuity to a $4 \mathrm{~m}$ test distance. For all analyses described below, disease severity was defined by the FDACL of the worse eye.

Disease asymmetry was defined for continuous variables by taking the absolute difference between eyes in each particular outcome (visual acuity, flat keratometry (K), steep $\mathrm{K}$, corneal toricity, spherical equivalent of the manifest refraction, and the cylindrical component of the manifest refraction). Asymmetry in corneal toricity was defined as follows:

(LE steep K-LE flat K)-(RE steep K-RE flat $\mathrm{K}$ )

Pearson's correlation coefficients were used to examine the relation between the continuous variables (asymmetry) and FDACL (severity). Subgroup analyses were performed whereby patients were placed into a mild/moderate or severe group based on the FDACL outcome associated with their worse eye $($ FDACL $\leqslant 52 \mathrm{D}=$ mild/moderate subgroup and
FDACL $>52$ D = severe subgroup). Pearson's correlation coefficients were then used to examine the relation between the clinical markers of asymmetry and severity within these subgroups, and subgroup specific p values are reported. A Fisher's Z transformation was used to compare correlations between these two subgroups, and p values are reported for these comparisons. In general, given our large sample size, we had $80 \%$ power to find a correlation coefficient of 0.14 to be highly statistically significant $(\mathrm{p}<0.0001)$. Therefore, we recommend evaluating the magnitude of the reported correlation coefficient using the following guidelines: a correlation of 0.1 to 0.3 is small, 0.3 to 0.5 is moderate, and greater than 0.5 is large. $^{12}$

For categorical variables (Vogt's striae, Fleischer's ring, and corneal scarring), disease asymmetry was defined by the presence of the outcome in neither, one, or both eyes. Disease severity was again defined using the FDACL value from the worse (steeper) eye. One way analysis of variance (ANOVA) was used to examine differences in the average FDACL by the presence of a categorical clinical sign (Vogt's striae, Fleischer's ring, and corneal scarring) in two, one, and no eyes. When the overall ANOVA p value was significant, Tukey's HSD test was used for pairwise comparisons. Outcomes were considered significantly different at $p \leqslant 0.05$. Additionally, ANOVA was used to test the interaction of FDACL severity subgroup (mild/moderate and severe) by the number of eyes with the categorical sign. When this interaction was significant, the regression equation for the prediction of FDACL by the number of eyes with the sign is reported separately by FDACL severity group. Proc Syslin in SAS was used to test the equality of the slope of the two regression equations.

\section{RESULTS}

A total of 1037 participants were eligible for these analyses. The mean age of this sample is 38.9 (standard deviation, SD 10.9) years, of which $582(56 \%)$ were male and 455 (44\%) were female. Patients were not eligible for the following reasons: 116 patients had undergone penetrating keratoplasty, two patients had undergone epikeratoplasty, 12 patients wore their monovision correction to the baseline visit, and 42 patients had at least one missing FDACL value.

Table 1 presents the mean differences between eyes for visual acuity, manifest refraction (spherical equivalent and cylinder), flat keratometry, steep keratometry, and corneal cylinder outcomes and their correlations with the FDACL of the worse eye. For these analyses, partial correlations were also determined to remove any potential effects of age, and no differences were observed in the results (not presented). As can be seen, there were small correlations between asymmetry in visual acuity and disease severity, and more moderate correlations between asymmetry in keratometry and disease severity and asymmetry in refraction and disease severity.

Table 2 presents subgroup analyses for the continuous clinical variables. Many of the correlations of between-eye asymmetry and severity remain small to moderate; however, a general trend can be seen whereby the correlation between asymmetry and severity in the more severe group is stronger than that observed in the mild/moderate group.

Table 3 presents the results for the categorical outcomes. Again, age was included in these models as a covariate, and no differences were found in the results (data not presented). For Vogt's striae, those with one eye (54.10 (SD 5.46) D) or two eyes (54.78 (SD 5.53) D) affected had significantly higher FDACL means than those with neither eye affected (51.83 (SD 5.13) D). For Fleischer's ring, the mean FDACL for those with no eyes affected was 51.15 (SD 5.63) D, with one eye affected was 51.87 (SD 4.85) D, and with both eyes 
Table 1 Association of the absolute difference in visual acuity, refraction, and keratometry between eyes with the FDACL of the worse eye (all $p<0.0001$ )

\begin{tabular}{|c|c|c|c|}
\hline Clinical measure & $\begin{array}{l}\text { Sample } \\
\text { Size }\end{array}$ & $\begin{array}{l}\text { Mean (SD) } \\
\text { difference } \\
\text { between eyes }\end{array}$ & $\begin{array}{l}\text { Correlation } \\
\text { with FDACL } \\
\text { (worse eye) }\end{array}$ \\
\hline $\begin{array}{l}\text { High contrast entrance } \\
\text { visual acuity (letters } \\
\text { correct) }\end{array}$ & 1035 & $11.38(13.77)$ & 0.25 \\
\hline $\begin{array}{l}\text { Low contrast entrance } \\
\text { visual acuity (letters } \\
\text { correct) }\end{array}$ & 1032 & $12.54(13.62)$ & 0.25 \\
\hline $\begin{array}{l}\text { High contrast best } \\
\text { corrected visual acuity } \\
\text { (letters correct) }\end{array}$ & 1028 & $7.58(8.00)$ & 0.28 \\
\hline $\begin{array}{l}\text { Low contrast best } \\
\text { corrected visual acuity } \\
\text { (letters correct) }\end{array}$ & 1017 & $9.38(9.68)$ & 0.28 \\
\hline $\begin{array}{l}\text { Manifest refraction- } \\
\text { spherical equivalent (D) }\end{array}$ & 1034 & 3.07 (3.73) & 0.41 \\
\hline $\begin{array}{l}\text { Manifest refraction- } \\
\text { cylinder (D) }\end{array}$ & 1034 & $1.54(1.41)$ & 0.13 \\
\hline Flat keratometry (D) & 1030 & $3.39(4.13)$ & 0.61 \\
\hline Steep keratometry (D) & 1028 & $4.19(4.17)$ & 0.54 \\
\hline Corneal toricity (D) & 1027 & $2.09(2.12)$ & 0.23 \\
\hline
\end{tabular}

affected was 53.11 (SD 5.45) D, which were all statistically different from each other.

For corneal scarring, the mean FDACL for those with either one or both eyes affected (56.48 (SD 5.80) D and 56.72 (SD 5.74) D, respectively) differed significantly from those with no eyes affected (50.92 (SD 4.10) D). After testing the interaction of FDACL severity group and the number of eyes with scarring, the effect of the number of eyes with scarring was found to be inconsistent across FDACL severity groups (ANOVA for interaction term, $\mathrm{p}<0.0001$ ). For the severe FDACL group, FDACL steepened as the number of eyes with scarring increased (0 eyes, 55.54 (SD 2.91) D; l eye, 58.93 (SD 4.62) D; 2 eyes 59.03 (SD 4.30) D). For the mild/ moderate group, FDACL values remained relatively constant

Table 2 Association of the absolute difference between eyes on clinical measures with the FDACL of the worse eye, by FDACL severity group

\begin{tabular}{|c|c|c|c|c|}
\hline \multirow[b]{2}{*}{ Clinical measure* } & \multirow{2}{*}{$\begin{array}{l}\text { FDACL severity } \\
\text { groupt }\end{array}$} & \multicolumn{2}{|c|}{$\begin{array}{l}\text { Association } \\
\text { with FDACL } \\
\text { of worse eye }\end{array}$} & \multirow{2}{*}{$\begin{array}{l}\text { p Value } \\
\text { comparing } \\
\text { correlations }\end{array}$} \\
\hline & & $r$ & p Value & \\
\hline \multirow{14}{*}{$\begin{array}{l}\text { High contrast } \\
\text { entrance visual acuity } \\
\text { Low contrast } \\
\text { entrance visual acuity } \\
\text { High contrast best } \\
\text { corrected visual acuity } \\
\text { Low contrast best } \\
\text { corrected visual acuity } \\
\text { Manifest refraction: } \\
\text { spherical equivalent } \\
\text { Manifest refraction: } \\
\text { cylinder } \\
\text { Flat keratometry }\end{array}$} & Mild/moderate & 0.10 & 0.02 & \multirow[t]{2}{*}{0.33} \\
\hline & Severe & 0.16 & 0.0004 & \\
\hline & Mild/moderate & 0.11 & 0.009 & \multirow[t]{2}{*}{0.44} \\
\hline & Severe & 0.16 & 0.0005 & \\
\hline & Mild/moderate & 0.10 & 0.02 & \multirow{2}{*}{0.02} \\
\hline & Severe & 0.24 & $<0.0001$ & \\
\hline & Mild/moderate & 0.13 & 0.003 & \multirow[t]{2}{*}{0.24} \\
\hline & Severe & 0.20 & $<0.0001$ & \\
\hline & Mild/moderate & 0.19 & $<0.0001$ & \multirow[t]{2}{*}{0.04} \\
\hline & Severe & 0.31 & $<0.0001$ & \\
\hline & Mild/moderate & 0.05 & 0.26 & \multirow[t]{2}{*}{0.95} \\
\hline & Severe & 0.04 & 0.34 & \\
\hline & Mild/moderate & 0.36 & $<0.0001$ & \multirow[t]{2}{*}{0.01} \\
\hline & Severe & 0.50 & $<0.0001$ & \\
\hline \multirow{2}{*}{ Steep keratometry } & Mild/moderate & 0.36 & $<0.0001$ & \multirow[t]{2}{*}{0.85} \\
\hline & Severe & 0.37 & $<0.0001$ & \\
\hline \multirow[t]{2}{*}{ Corneal toricity } & Mild/moderate & 0.28 & $<0.0001$ & \multirow[t]{2}{*}{0.0002} \\
\hline & Severe & 0.06 & 0.23 & \\
\hline
\end{tabular}

* Represent the absolute differences between eyes. tFDACL severity is based on the FDACL of the worse eye where mild/ moderate $=$ FDACL(D) $\leqslant 52(n=557)$ and severe $=F D A C L(D)>52$ $(n=480)$.
Table 3 Analysis of variance models for categorical clinical signs

\begin{tabular}{lll}
\hline Categorical asymmetry sign & Mean (SD) FDACL, D & Overall p value* \\
\hline Vogt's striae & $51.83(5.13)^{a}$ & \\
0 eyes $(n=658)$ & $54.10(5.46)^{b}$ & \\
1 eye $(n=277)$ & $54.78(5.53)^{b}$ & \\
2 eyes $(n=101)$ & $51.15(5.63)^{a}$ & 0.0004 \\
Fleischer's ring & $51.87(4.85)^{b}$ & \\
0 eyes $(n=76)$ & $53.11(5.45)^{c}$ & \\
1 eye $(n=200)$ & $50.92(4.10)^{a}$ & $<0.0001$ \\
2 eyes $(n=760)$ & $56.48(5.80)^{b}$ \\
Corneal scarring & $56.72(5.74)^{b}$ \\
0 eyes $(n=705)$ & \\
1 eye $(n=205)$ & \\
2 eyes $(n=126)$ & \\
\hline \multirow{2}{*}{ Overall $p$ value based on ANOVA. Tukey's HSD used for pairwise } \\
group comparisons. Means with different letters are significantly different \\
( $p \leqslant 0.05)$.
\end{tabular}

for participants with 0,1 , or 2 scarred eyes (48.65 (SD 2.31) D, 49.64 (SD 2.03) D, and 49.00 (SD 1.79) D, respectively). To illustrate the differential effect of the number of scarred eyes, a linear regression model was created for each FDACL severity group showing the prediction of FDACL by the number of eyes with scarring (fig 1). The slopes of the two regression lines were significantly different $(p<0.0001)$. No differential effects were present across severity groups for the Vogt's striae and Fleischer's ring outcomes (data not reported).

\section{DISCUSSION}

In a previous report, we showed that the degree of asymmetry in clinical outcomes associated with keratoconus is large compared with a group of "normal" myopic subjects. ${ }^{6}$ In this report, we have shown that for many of these same outcomes, the degree of asymmetry is associated with disease severity. For flat keratometry, high contrast, best corrected visual acuity, spherical equivalent, and corneal scarring the results further indicated that the association between disease asymmetry and severity was even greater in patients with more severe keratoconus than in patients with mild/moderate keratoconus. One might conclude from these results that as patients proceed through the disease process (presumably becoming more severe), more marked asymmetry in the disease also occurs. This relation will be better described as the longitudinal data associated with the study become available.

Our results show that the relation between asymmetry and severity is most robust in outcomes relating to corneal curvature, refractive error, and corneal scarring. This is an important finding as these outcomes (particularly corneal

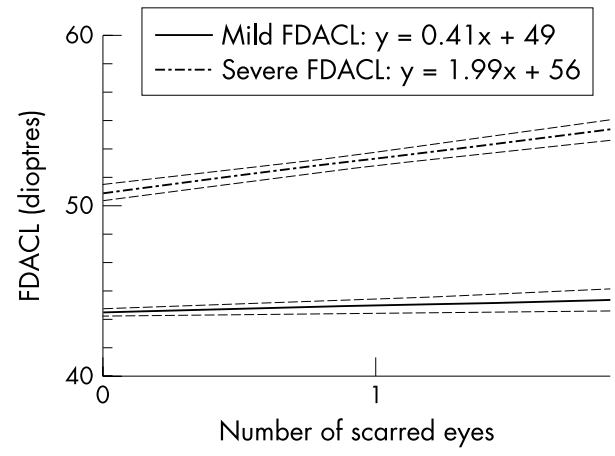

Figure 1 FDACL (D) and the number of scarred eyes by FDACL severity group. Regression lines are presented with $95 \%$ confidence intervals. 
Table 4 Association between flat keratometry, steep keratometry, and corneal toricity with the FDACL overall and by FDACL severity group

\begin{tabular}{|c|c|c|c|c|}
\hline \multirow[b]{2}{*}{ Clinical measure* } & \multirow[b]{2}{*}{ FDACL severity group† } & \multicolumn{2}{|c|}{$\begin{array}{l}\text { Association with FDACL } \\
\text { of worse eye }\end{array}$} & \multirow{2}{*}{$\begin{array}{l}\text { p Value comparing } \\
\text { correlations }\end{array}$} \\
\hline & & $r$ & $\mathrm{p}$ Value & \\
\hline \multirow[t]{2}{*}{ Flat keratometry } & $\begin{array}{l}\text { Overall } \\
\text { Mild/moderate }\end{array}$ & 0.82 & $<0.0001$ & \multirow[b]{2}{*}{0.0004} \\
\hline & $\begin{array}{l}\text { Mild/moderate } \\
\text { Severe }\end{array}$ & 0.71 & $<0.0001$ & \\
\hline \multirow[t]{3}{*}{ Steep keratometry } & Overall & 0.86 & $<0.0001$ & \multirow{3}{*}{0.01} \\
\hline & Mild/moderate & 0.66 & $<0.0001$ & \\
\hline & Severe & 0.74 & $<0.0001$ & \\
\hline \multirow[t]{3}{*}{ Corneal toricity } & Overall & 0.23 & $<0.0001$ & \multirow{3}{*}{$<0.0001$} \\
\hline & Mild/moderate & 0.32 & $<0.0001$ & \\
\hline & Severe & 0.01 & 0.87 & \\
\hline
\end{tabular}

curvature and scarring) are likely to be directly related to the clinical care of these patients (for example, contact lens fitting and surgery). For instance, corneal scarring affects visual function, and both corneal scarring and vision are primary determinants of the need for penetrating keratoplasty in keratoconus. ${ }^{5}$ Of the sample of patients described here who had not had a penetrating keratoplasty before the baseline visit $(n=1,037), 103$ have since gone on to penetrating keratoplasty during the course of follow up. Of these, $79(77 \%)$ had a unilateral transplant, and $24(23 \%)$ had bilateral transplants $\left(\chi^{2}, \mathrm{p}<0.0001\right)$.

Interestingly, our subgroup analyses show that the strength of the association between disease asymmetry and severity differed in mild/moderate and severe keratoconus for flat keratometry but not for steep keratometry. Another interesting finding is the difference between the mild/ moderate and severe groups in terms of their respective relations between asymmetry and severity in corneal toricity. In order to help explain this finding, we conducted some post hoc correlation analyses of the relation between flat keratometry, steep keratometry, and corneal toricity (all of the worse eye) and FDACL (of the worse eye). These results are presented in table 4 . Both flat and steep keratometry are highly correlated with FDACL $(r=0.82$ and 0.86 respectively), yet corneal toricity is much less correlated with FDACL $(r=0.23)$. For both flat and steep keratometry, the relation between the outcome variable and FDACL is stronger for patients with severe disease compared with patients with mild/moderate disease. However, this is not true for corneal toricity, where there is a moderate correlation between toricity and FDACL for patients with mild/moderate disease $(r=0.32)$ and no correlation between toricity and FDACL for patients with severe disease $(r=0.01)$. This may indicate that in earlier stages of keratoconus, the cornea increases in toricity until it reaches some threshold level after which it stabilises throughout the remainder of disease progression. It may also indicate that FDACL changes are independent of toricity changes.

We did find smaller, but statistically significant, correlations between the absolute difference in visual acuity outcomes and disease severity. Again, these analyses were highly powered to find small correlations statistically significant. These smaller correlations found in visual outcomes probably reflect the fact that we analysed asymmetry associated with corrected visual acuity rather than uncorrected visual acuity. In that regard, we "masked" the true asymmetry in vision associated with keratoconus, unlike our measures of refractive error and corneal curvature. Although this is true, we believe that the fact that all correlations in this study were in the same direction-that is, there was a positive correlation in all analyses-further supports the notion that disease asymmetry and severity are substantially related in keratoconus.

\section{CONCLUSIONS}

Patients with keratoconus have notable between-eye asymmetry, and the degree of asymmetry is greater in cases of more severe disease. This is reflected in outcomes related to the clinical care of these patients such as penetrating keratoplasty.

\section{ACKNOWLEDGEMENTS}

The Collaborative Longitudinal Evaluation of Keratoconus (CLEK) Study is supported by the National Eye Institute/National Institutes of Health, grants EY10419, EY10069, EY10077, EY12656, and EY02687. It also was supported by Conforma Contact Lenses, Paragon Vision Sciences, CIBA Vision Corporation, and the Ohio Lions Eye Research Foundation.

\section{Authors' affiliations}

J J Nichols, K Zadnik, Ohio State University, College of Optometry, Columbus, $\mathrm{OH}$, USA

K Steger-May, Division of Biostatistics, Washington University School of Medicine, St. Louis, MO, USA

T B Edrington, Southern California College of Optometry, Fullerton, CA, USA

\section{REFERENCES}

1 Macsai MS, Varley GA, Krachmer JH. Development of keratoconus after contact lens wear. Patient characteristics. Arch Ophthalmol 1990;108:534-8.

2 Zadnik K, Barr JT, Gordon MO, et al. Biomicroscopic signs and disease severity in keratoconus. Cornea 1996;15:139-46.

3 Tuft SJ, Moodaley LC, Gregory WM, et al. Prognostic factors for the progression of keratoconus. Ophthalmology 1994;101:439-47.

4 Ihalainen A. Clinical and epidemiological features of keratoconus genetic and external factors in the pathogenesis of the disease. Acta Ophthalmol Suppl 1986; 178:1-64.

5 Lass JH, Lembach RG, Park SB, et al. Clinical management of keratoconus. A multicenter analysis. Ophthalmology 1990;97:433-45.

6 Zadnik K, Steger-May K, Fink BA, et al. Between-eye asymmetry in keratoconus. Cornea 2002;21:671-9.

7 Edrington TB, Szczotka LB, Begley CG, et al. Repeatability and agreement of two corneal-curvature assessments in keratoconus: keratometry and the first definite apical clearance lens (FDACL). Cornea 1998;17:267-77.

8 Davis L, Schechtman KB, Begley CG, et al. Repeatability of refraction and corrected visual acuity in keratoconus. The CLEK Study Group. Collaborative Longitudinal Evaluation of Keratoconus. Optom Vis Sci 1998;75:887-96.

9 Gordon MO, Schechtman KB, Davis L, et al. Visual acuity repeatability in keratoconus: impact on sample size. Optom Vis Sci 1998;75:249-57.

10 Zadnik K, Barr JT, Edrington TB, et al. Baseline findings in the Collaborative Longitudinal Evaluation of Keratoconus (CLEK) Study. Invest Ophthalmol Vis Sci 1998;39:2537-46.

11 Mandell RB. Contact lens practice. 4th ed. Springfield, IL: Charles C Thomas; 1988.

12 Cohen J. Statistical power analysis for the behavioral sciences. 2nd ed. Hillsdale, NJ: L Erlbaum Associates, 1988. 